\title{
Coupled flow-polymer dynamics via statistical field theory: modeling and computation
}

\author{
Hector D. Ceniceros ${ }^{1}$ Glenn H. Fredrickson ${ }^{2}$ \\ George O. Mohler ${ }^{3, *}$
}

\begin{abstract}
Field-theoretic models, which replace interactions between polymers with interactions between polymers and one or more conjugate fields, offer a systematic framework for coarse-graining of complex fluids systems. While this approach has been used successfully to investigate a wide range of polymer formulations at equilibrium, field-theoretic models often fail to accurately capture the non-equilibrium behavior of polymers, especially in the early stages of phase separation. Here the "two-fluid" approach serves as a useful alternative, treating the motions of fluid components separately in order to incorporate asymmetries between polymer molecules. In this work we focus on the connection of these two theories, drawing upon the strengths of each of the approaches in order to couple polymer microstructure with the dynamics of the flow in a systematic way. For illustrative purposes we work with an inhomogeneous melt of elastic dumbbell polymers, though our methodology will apply more generally to a wide variety of inhomogeneous systems. First we derive the model, incorporating thermodynamic forces into a two-fluid model for the flow through the introduction of conjugate chemical potential and elastic strain fields for the polymer density and stress. The resulting equations are composed of a system of fourth order PDEs coupled with a nonlinear, nonlocal optimization problem to determine the conjugate fields. The coupled system is severely stiff and with a high degree of computational complexity. Next, we overcome the formidable numerical challenges posed by the model by designing a robust semi-implicit method based on linear asymptotic behavior of the leading order terms at small scales, by exploiting the exponential structure of global (integral) operators, and by parallelizing the nonlinear optimization problem. The semi-implicit method effectively removes the fourth order stability constraint associated with explicit methods and we observe only a first order time-step restriction. The algorithm for solving the nonlinear optimization problem, which takes advantage of the form of the operators being optimized, reduces the overall computational cost of simulations by several orders of magnitude. We illustrate the methodology with several examples of phase separation in an initially quiescent flow.
\end{abstract}

Key words: inhomogeneous polymer blends, polymer field theory, two-fluid model, semi-implicit methods, complex fluids, soft materials 


\section{Introduction}

Self-assembled polymeric systems are of fundamental importance in a variety of applications, such as multiphase plastic materials and solution formulations, and recently a great deal of interest has focused on the application of polymers in soft material nanontechnology [8]. Though experimental methods are vital in the design of such materials, computer simulation and theory can aid in the exploration of parameter spaces, reducing both the time and cost of the material design process.

One approach that has been very useful in the study of self-assembly in polymeric systems is polymer field theory. Field-theoretic models, which replace interactions between polymers with interactions between polymers and one or more conjugate fields, allow for systematic coarse-graining and thus simulation is possible over a broad range of scales [8]. Such methods have found wide application in the study of the equilibrium structure and thermodynamics of inhomogeneous polymer melts and concentrated solutions of homo-, block, and graft copolymers $[6,8,10,12,14-16]$. However, flow dynamics are often neglected in such models and polymer field theory has been less successful in the study of non-equilibrium regimes, such as the initial stages of phase separation.

For polymeric systems in which flow dynamics of component molecules play a significant role, the "two-fluid" approach $[5,17,18]$ has proven to be a useful formalism. Two-fluid models treat the motion of fluid components separately and have provided insight into polymeric fluids with asymmetries between component molecules. The drawback of such models, in comparison to polymer field theory, is that the microscopic structure of polymer molecules is not easily incorporated and phenomenological equations are typically used for the chemical potentials and polymer stresses.

In this work we focus on the connection of these two theories, incorporating polymer microstructure, via field theory, into the two-fluid modeling approach. To our knowledge we present the first computational study of coupled flowpolymer dynamics under the framework developed in [7], avoiding constitutive laws for the chemical potential fields and polymer stresses. For illustrative purposes we work with an inhomogeneous melt of elastic dumbbell polymers,

\footnotetext{
* Corresponding author.

1 Department of Mathematics, University of California, Santa Barbara, CA 931065080 (hdc@math.ucsb.edu).

2 Department of Chemical Engineering and Materials Research Laboratory, University of California, Santa Barbara, CA 93106-5080 (ghf@mrl.ucsb.edu).

3 Department of Mathematics, University of California, Santa Barbara, CA 931065080 (georgemohler@gmail.com).
} 
though our methodology will apply more generally to a wide variety of inhomogeneous systems. We thus view this work as a stepping stone, providing a foundation for more advanced studies of polymer dynamics.

Our methodology is based on the work in [7], where a model for polymer solutions is developed that embeds exact thermodynamic forces, through the introduction of conjugate chemical potential and elastic strain fields for the polymer density and stress variables, into a two-fluid model for the flow. We note that a similar model is considered in [9], where the two-fluid approach is combined with polymer field-theory for inhomogeneous polymer melts and a numerical method is presented. However, the model in [9] is considerably simplified from [7] in that only chemical potential fields are introduced and a phenomenological constitutive law for stress dynamics is used.

Due to the complex nature of the models that arise from the approach developed in [7], several computational challenges must be overcome. The form of the models is a system of high order, nonlinear partial differential equations for the density, stress, and velocity variables, coupled with a nonlinear, nonlocal optimization problem for the chemical potential and elastic strain variables. The presence of high order derivatives leads to numerical stiffness and the size of the discrete time-step used in simulation is severely restricted for explicit methods. Additionally, the nonlinear optimization problem must be solved at each point in time and thus the computational cost of each time-step is much higher than is typically the case in standard numerical CFD problems. In this work we address these computational challenges and develop a numerical methodology capable of efficiently simulating polymeric fluids modeled by the approach in [7].

The organization of the paper is as follows. In Section 2, we derive a model for an inhomogeneous melt of elastic dumbbell polymers, connecting the microscopic description of the polymers to the macroscopic description of the fluid. The model is composed of a system of fourth order PDEs coupled with a nonlinear optimization problem for the conjugate fields. In Section 3, we present the numerical method for the model, which consists of a semi-implicit time integration scheme for the system of PDEs coupled with an efficient, parallel algorithm for the nonlinear optimization problem. In designing the semiimplict integration scheme, we draw upon ideas used for phase-field models of polymers $[1,3]$ and linear operators are extracted from the right hand side of the PDEs and treated implicitly. Whereas explicit methods have a fourth order time-step restriction, we observe only a first order stability constraint for the semi-implicit strategy. The algorithm for the nonlinear optimization problem, which includes techniques designed for problems arising in polymer self-consistent field theory [2], takes advantage of the specific form of the operators being optimized and reduces the computational cost of simulation by several orders of magnitude. In Section 4, we implement our methodology for 
a phase separating melt of elastic dumbbells and also investigate the effects of asymmetry between the fluid components. We provide an estimate of the timestep restriction of the semi-implicit method and highlight the computational savings over alternative methodologies.

\section{Derivation of the model}

\subsection{Microscopic model for elastic dumbbell polymers}

We start with a particle based model of a binary, incompressible melt of elastic dumbbells (a bead-spring model for the polymers using two beads). We let $\mathbf{R}_{\alpha_{1}}$ and $\mathbf{R}_{\alpha_{2}}$ denote the positions of the 1 st and 2 nd beads of the $\alpha$ th polymer of type $a$ and $\mathbf{R}_{\beta_{1}}$ and $\mathbf{R}_{\beta_{2}}$ denote the positions of the 1 st and 2 nd beads of the $\beta$ th polymer of type $b$. Here $\alpha=1, \ldots, n_{a}$ and $\beta=1, \ldots, n_{b}$, where $n_{a}$ and $n_{b}$ are the number of polymers of type $a$ and $b$.

We work in the $n V T$ canonical ensemble and conformations of noninteracting polymers are given a Gaussian statistical weight, $\exp \left(-U_{0} / k_{B} T\right)$, with a stretching free energy of the form,

$$
U_{0}=\frac{k T}{4 R g_{a}^{2}} \sum_{\alpha=1}^{n_{a}}\left(\mathbf{R}_{\alpha_{2}}-\mathbf{R}_{\alpha_{1}}\right)^{2}+\frac{k T}{4 R g_{b}^{2}} \sum_{\beta=1}^{n_{b}}\left(\mathbf{R}_{\beta_{2}}-\mathbf{R}_{\beta_{1}}\right)^{2}
$$

where $R g_{a}^{2}$ and $R g_{b}^{2}$ are the unperturbed radii-of-gyration, $k_{B}$ is the Boltzmann constant, and $T$ is the thermodynamic temperature of the system. To simplify the notation we express energies in units of $k_{B} T$ and set $k_{B} T=1$.

The effective potential energy function representing nonbonded interactions between monomers (beads) on the same polymer and between monomers on different polymers is given by

$$
U_{1}=\int d \mathbf{r}\left[\chi_{1} \hat{\rho}_{a}(\mathbf{r}) \hat{\rho}_{b}(\mathbf{r})+\frac{1}{2} \chi_{2}\left|\nabla \hat{\rho}_{a}(\mathbf{r})\right|^{2}+\frac{1}{2} \chi_{2}\left|\nabla \hat{\rho}_{b}(\mathbf{r})\right|^{2}\right]
$$

where $\hat{\rho}_{a}(\mathbf{r})=\sum_{i=1}^{2} \sum_{\alpha=1}^{n_{a}} \delta\left(\mathbf{r}-\mathbf{R}_{\alpha_{i}}\right)$ and $\hat{\rho}_{b}(\mathbf{r})=\sum_{i=1}^{2} \sum_{\beta=1}^{n_{b}} \delta\left(\mathbf{r}-\mathbf{R}_{\beta_{i}}\right)$ are microscopic monomer number densities and $\chi_{1}$ and $\chi_{2}$ are energetic parameters that penalize $a-b$ bead contacts and density gradients, respectively. Additionally, we have expressions for the microscopic elastic stresses [4] that take the form 


$$
\begin{array}{r}
{\left[\hat{\boldsymbol{\sigma}}_{a}(\mathbf{r})\right]_{j k}=\frac{1}{4 R g_{a}^{2}} \sum_{\alpha=1}^{n_{a}}\left(\mathbf{R}_{\alpha_{2}}-\mathbf{R}_{\alpha_{1}}\right)_{j}\left(\mathbf{R}_{\alpha_{2}}-\mathbf{R}_{\alpha_{1}}\right)_{k} \times} \\
{\left[\delta\left(\mathbf{r}-\mathbf{R}_{\alpha_{1}}\right)+\delta\left(\mathbf{r}-\mathbf{R}_{\alpha_{2}}\right)\right]}
\end{array}
$$

and

$$
\begin{gathered}
{\left[\hat{\boldsymbol{\sigma}}_{b}(\mathbf{r})\right]_{j k}=\frac{1}{4 R g_{b}^{2}} \sum_{\beta=1}^{n_{b}}\left(\mathbf{R}_{\beta_{2}}-\mathbf{R}_{\beta_{1}}\right)_{j}\left(\mathbf{R}_{\beta_{2}}-\mathbf{R}_{\beta_{1}}\right)_{k} \times} \\
{\left[\delta\left(\mathbf{r}-\mathbf{R}_{\beta_{1}}\right)+\delta\left(\mathbf{r}-\mathbf{R}_{\beta_{2}}\right)\right] .}
\end{gathered}
$$

With this microscopic description of the polymers, the configurational partition function is given as

$$
Z=\prod_{\alpha=1}^{n_{a}} \prod_{\beta=1}^{n_{b}} \int d \mathbf{R}_{\alpha_{1}} \int d \mathbf{R}_{\alpha_{2}} \int d \mathbf{R}_{\beta_{1}} \int d \mathbf{R}_{\beta_{2}} \exp \left(-U_{0}-U_{1}\right)
$$

\subsection{Field-theoretic form of the model}

To transform the microscopic model into a field theory, we first insert the identity

$$
\begin{aligned}
\int \mathcal{D}\left[\rho_{a}\right] \int \mathcal{D}\left[\boldsymbol{\sigma}_{a}\right] & \delta\left(\rho_{a}-\hat{\rho}_{a}\right) \delta\left(\boldsymbol{\sigma}_{a}-\hat{\boldsymbol{\sigma}}_{a}\right) \\
& =\int \mathcal{D}\left[\rho_{b}\right] \int \mathcal{D}\left[\boldsymbol{\sigma}_{b}\right] \delta\left(\rho_{b}-\hat{\rho}_{b}\right) \delta\left(\boldsymbol{\sigma}_{b}-\hat{\boldsymbol{\sigma}}_{b}\right)=1
\end{aligned}
$$

into the expression for the configurational partition function given by Equation (5), where the integrals appearing in this equation are functional integrals [8] over the real fields $\rho_{a}, \rho_{b}, \boldsymbol{\sigma}_{a}$ and $\boldsymbol{\sigma}_{b}$. Next we introduce functional integrals over the conjugate chemical potential fields $\mu_{a}(\mathbf{r})$ and $\mu_{b}(\mathbf{r})$ and elastic strain fields $\boldsymbol{\epsilon}_{a}(\mathbf{r})$ and $\boldsymbol{\epsilon}_{b}(\mathbf{r})$ through the identities

$$
\begin{aligned}
& \delta\left(\rho_{a}-\hat{\rho}_{a}\right) \delta\left(\boldsymbol{\sigma}_{a}-\hat{\boldsymbol{\sigma}}_{a}\right) \\
& \quad=\int \mathcal{D}\left[\mu_{a}\right] \int \mathcal{D}\left[\boldsymbol{\epsilon}_{a}\right] \exp \left(i \int d \mathbf{r}\left[\mu_{a}\left(\rho_{a}-\hat{\rho}_{a}\right)+\boldsymbol{\epsilon}_{a}:\left(\boldsymbol{\sigma}_{a}-\hat{\boldsymbol{\sigma}}_{a}\right)\right]\right), \\
& \delta\left(\rho_{b}-\hat{\rho}_{b}\right) \delta\left(\boldsymbol{\sigma}_{b}-\hat{\boldsymbol{\sigma}}_{b}\right) \\
& \quad=\int \mathcal{D}\left[\mu_{b}\right] \int \mathcal{D}\left[\boldsymbol{\epsilon}_{b}\right] \exp \left(i \int d \mathbf{r}\left[\mu_{b}\left(\rho_{b}-\hat{\rho}_{b}\right)+\boldsymbol{\epsilon}_{b}:\left(\boldsymbol{\sigma}_{b}-\hat{\boldsymbol{\sigma}}_{b}\right)\right]\right),
\end{aligned}
$$


where $\boldsymbol{\epsilon}: \boldsymbol{\sigma}=\sum_{i j} \epsilon_{i j} \sigma_{i j}$. These two steps allow the configurational partition function to be expressed as

$$
\begin{aligned}
Z= & \int \mathcal{D}\left[\rho_{a}\right] \int \mathcal{D}\left[\rho_{b}\right] \int \mathcal{D}\left[\boldsymbol{\sigma}_{a}\right] \int \mathcal{D}\left[\boldsymbol{\sigma}_{b}\right] \int \mathcal{D}\left[\mu_{a}\right] \int \mathcal{D}\left[\mu_{b}\right] \int \mathcal{D}\left[\boldsymbol{\epsilon}_{a}\right] \int \mathcal{D}\left[\boldsymbol{\epsilon}_{b}\right] \\
& \times \exp \left(-H\left[\rho_{a}, \rho_{b}, \boldsymbol{\sigma}_{a}, \boldsymbol{\sigma}_{b}, \mu_{a}, \mu_{b}, \boldsymbol{\epsilon}_{a}, \boldsymbol{\epsilon}_{b}\right]\right)
\end{aligned}
$$

where the effective Hamiltonian $H$ in this equation is given by

$$
\begin{aligned}
H=\int d \mathbf{r} & {\left[\chi_{1} \rho_{a}(\mathbf{r}) \rho_{b}(\mathbf{r})+\frac{1}{2} \chi_{2}\left|\nabla \rho_{a}(\mathbf{r})\right|^{2}+\frac{1}{2} \chi_{2}\left|\nabla \rho_{b}(\mathbf{r})\right|^{2}\right.} \\
& \left.-i \mu_{a}(\mathbf{r}) \rho_{a}(\mathbf{r})-i \mu_{b}(\mathbf{r}) \rho_{b}(\mathbf{r})-i \boldsymbol{\epsilon}_{a}(\mathbf{r}): \boldsymbol{\sigma}_{a}(\mathbf{r})-i \boldsymbol{\epsilon}_{b}(\mathbf{r}): \boldsymbol{\sigma}_{b}(\mathbf{r})\right] \\
& -n_{a} \ln Q_{a}\left[i \mu_{a}, i \boldsymbol{\epsilon}_{a}\right]-n_{a} \ln Q_{b}\left[i \mu_{b}, i \boldsymbol{\epsilon}_{b}\right] .
\end{aligned}
$$

The functionals $Q_{a}$ and $Q_{b}$, which are partition functions of a single dumbbell polymer in the purely imaginary chemical potential and strain fields [8], satisfy

$$
\begin{aligned}
Q_{a}=\frac{1}{Q_{a 0}} \int d \mathbf{r} \int d \mathbf{r}^{\prime} \exp ( & -i \mu_{a}(\mathbf{r})-i \mu_{a}\left(\mathbf{r}^{\prime}\right) \\
& \left.-\frac{1}{4 R g_{a}^{2}}\left(\mathbf{r}-\mathbf{r}^{\prime}\right)^{2}:\left[I+i \boldsymbol{\epsilon}_{a}(\mathbf{r})+i \boldsymbol{\epsilon}_{a}\left(\mathbf{r}^{\prime}\right)\right]\right)
\end{aligned}
$$

and

$$
\begin{aligned}
Q_{b}=\frac{1}{Q_{b 0}} \int d \mathbf{r} \int d \mathbf{r}^{\prime} \exp ( & -i \mu_{b}(\mathbf{r})-i \mu_{b}\left(\mathbf{r}^{\prime}\right) \\
& \left.-\frac{1}{4 R g_{b}^{2}}\left(\mathbf{r}-\mathbf{r}^{\prime}\right)^{2}:\left[I+i \boldsymbol{\epsilon}_{b}(\mathbf{r})+i \boldsymbol{\epsilon}_{b}\left(\mathbf{r}^{\prime}\right)\right]\right)
\end{aligned}
$$

The normalization factors $Q_{a 0}$ and $Q_{b 0}$ are defined so that $Q_{a}[0,0]=Q_{b}[0,0]=$ 1 and in the above expression $I$ denotes the unit tensor.

\subsection{Mean-field approximation}

Thermodynamic forces are obtained through the variational derivatives of the effective Hamiltonian,

$$
\frac{\delta H}{\delta \rho_{a}}, \frac{\delta H}{\delta \rho_{b}}, \frac{\delta H}{\delta \boldsymbol{\sigma}_{a}}, \frac{\delta H}{\delta \boldsymbol{\sigma}_{b}}, \frac{\delta H}{\delta \mu_{a}}, \frac{\delta H}{\delta \mu_{b}}, \frac{\delta H}{\delta \boldsymbol{\epsilon}_{a}}, \frac{\delta H}{\delta \boldsymbol{\epsilon}_{b}} .
$$


For dense systems, such as the melt considered in this work, the functional integral appearing in Equation (9) is dominated by a saddle point, $\left(\mu_{a}^{*}, \mu_{b}^{*}, \boldsymbol{\epsilon}_{a}^{*}\right.$, $\left.\boldsymbol{\epsilon}_{b}^{*}\right)$, of $H[10]$, which satisfies:

$$
\begin{aligned}
& \left.\frac{\delta H}{\delta \mu_{a}}\right|_{\rho_{a}, \sigma_{a}, \mu_{a}=\mu_{a}^{*}, \epsilon_{a}=\epsilon_{a}^{*}}=\left.\frac{\delta H}{\delta \mu_{b}}\right|_{\rho_{b}, \sigma_{b}, \mu_{b}=\mu_{b}^{*}, \epsilon_{b}=\epsilon_{b}^{*}}=0, \\
& \left.\frac{\delta H}{\delta \boldsymbol{\epsilon}_{a}}\right|_{\rho_{a}, \sigma_{a}, \mu_{a}=\mu_{a}^{*}, \epsilon_{a}=\epsilon_{a}^{*}}=\left.\frac{\delta H}{\delta \epsilon_{b}}\right|_{\rho_{b}, \sigma_{b}, \mu_{b}=\mu_{b}^{*}, \epsilon_{b}=\epsilon_{b}^{*}}=0 .
\end{aligned}
$$

We thus ignore all fluctuations in the $\mu$ and $\epsilon$ fields and require Equations (14)(15) to hold. As it turns out, the physically relevant saddle points prove to be purely imaginary and for convenience we set $\mu_{a} \leftarrow i \mu_{a}, \mu_{b} \leftarrow i \mu_{b}, \boldsymbol{\epsilon}_{a} \leftarrow i \boldsymbol{\epsilon}_{a}$ and $\boldsymbol{\epsilon}_{b} \leftarrow i \boldsymbol{\epsilon}_{b}$. Thus for the remainder of this paper all fields will be purely real.

Taking the variational derivatives of $H$, we have

$$
\begin{aligned}
\frac{\delta H}{\delta \rho_{a}} & =\chi_{1} \rho_{b}-\chi_{2} \nabla^{2} \rho_{a}-\mu_{a} \\
\frac{\delta H}{\delta \boldsymbol{\sigma}_{a}} & =-\boldsymbol{\epsilon}_{a} \\
\frac{\delta H}{\delta \mu_{a}} & =\tilde{\rho}_{a}-\rho_{a} \\
\frac{\delta H}{\delta \boldsymbol{\epsilon}_{a}} & =\tilde{\boldsymbol{\sigma}}_{a}-\boldsymbol{\sigma}_{a}
\end{aligned}
$$

with similar equations holding for polymers of type $b$, where the density and stress operators $\tilde{\rho}_{a}$ and $\tilde{\boldsymbol{\sigma}}_{a}$ are given by

$$
\begin{aligned}
\tilde{\rho}_{a}(\mathbf{r}) & =-n_{a} \frac{\delta \ln Q_{a}}{\delta \mu_{a}} \\
& =\frac{2 n_{a}}{Q_{a} Q_{a 0}} \exp \left(-\mu_{a}(\mathbf{r})\right) \times \\
& \int d \mathbf{r}^{\prime} \exp \left(-\mu_{a}\left(\mathbf{r}^{\prime}\right)-\frac{1}{4 R g_{a}^{2}}\left(\mathbf{r}-\mathbf{r}^{\prime}\right)^{2}:\left[I+\boldsymbol{\epsilon}_{a}(\mathbf{r})+\boldsymbol{\epsilon}_{a}\left(\mathbf{r}^{\prime}\right)\right]\right)
\end{aligned}
$$

and

$$
\begin{aligned}
\tilde{\boldsymbol{\sigma}}_{a}(\mathbf{r}) & =-n_{a} \frac{\delta \ln Q_{a}}{\delta \boldsymbol{\epsilon}_{a}} \\
& =\frac{n_{a}}{2 R g_{a}^{2} Q_{a} Q_{a 0}} \exp \left(-\mu_{a}(\mathbf{r})\right) \times
\end{aligned}
$$




$$
\int d \mathbf{r}^{\prime}\left(\mathbf{r}-\mathbf{r}^{\prime}\right)^{2} \exp \left(-\mu_{a}\left(\mathbf{r}^{\prime}\right)-\frac{1}{4 R g_{a}^{2}}\left(\mathbf{r}-\mathbf{r}^{\prime}\right)^{2}:\left[I+\boldsymbol{\epsilon}_{a}(\mathbf{r})+\boldsymbol{\epsilon}_{a}\left(\mathbf{r}^{\prime}\right)\right]\right)
$$

\subsection{Two-fluid model}

To model the flow we adopt the two-fluid approach outlined in [5] and begin by introducing a Rayleigh functional, $\mathcal{R}=W / 2+\dot{H}$, which is the sum of half of the energy dissipation rate $W$ and the rate of change of the effective Hamiltonian $\dot{H}$. The model for $W$ assumes a local, frictional coupling associated with the relative motions of the polymers $a$ and $b$,

$$
W=\int d \mathbf{r} \xi\left|\mathbf{v}_{a}-\mathbf{v}_{b}\right|^{2}
$$

where

$$
\xi=\frac{\xi_{a} \xi_{b}}{\xi_{a}+\xi_{b}}
$$

$\xi_{a}=\xi_{a 0} \rho_{a}$ and $\xi_{b}=\xi_{b 0} \rho_{b}$ are polymer friction coefficients per unit volume, $\rho_{a}$ and $\rho_{b}$ are monomer number densities, and $\mathbf{v}_{a}$ and $\mathbf{v}_{b}$ are the species velocities of polymers $a$ and $b$.

The rate of change of the effective Hamiltonian is obtained using the chain rule:

$$
\dot{H}=\int d \mathbf{r}\left(\frac{\delta H}{\delta \rho_{a}} \frac{\partial \rho_{a}}{\partial t}+\frac{\delta H}{\delta \rho_{b}} \frac{\partial \rho_{b}}{\partial t}+\frac{\delta H}{\delta \boldsymbol{\sigma}_{a}}: \frac{\partial \boldsymbol{\sigma}_{a}}{\partial t}+\frac{\delta H}{\delta \boldsymbol{\sigma}_{b}}: \frac{\partial \boldsymbol{\sigma}_{b}}{\partial t}\right),
$$

where we have only retained the time variations of the fields that have microscopic Poisson-bracket couplings [11] to the polymer velocity fields.

Since $\hat{\rho}_{a}$ and $\hat{\rho}_{b}$ are microscopically conserved, the corresponding macroscopic density variables satisfy continuity equations:

$$
\frac{\partial \rho_{a}}{\partial t}=-\nabla \cdot\left(\mathbf{v}_{a} \rho_{a}\right)
$$

and

$$
\frac{\partial \rho_{b}}{\partial t}=-\nabla \cdot\left(\mathbf{v}_{b} \rho_{b}\right)
$$


The macroscopic stress variables corresponding to $\hat{\boldsymbol{\sigma}}_{a}$ and $\hat{\boldsymbol{\sigma}}_{b}$ are not conserved, but instead are rotated and advected by the polymer velocity according to:

$$
\frac{\partial \boldsymbol{\sigma}_{a}}{\partial t}=-\nabla \cdot\left(\mathbf{v}_{a} \boldsymbol{\sigma}_{a}\right)+\left(\nabla \mathbf{v}_{a}\right)^{T} \cdot \boldsymbol{\sigma}_{a}+\boldsymbol{\sigma}_{a} \cdot\left(\nabla \mathbf{v}_{a}\right)
$$

and

$$
\frac{\partial \boldsymbol{\sigma}_{b}}{\partial t}=-\nabla \cdot\left(\mathbf{v}_{b} \boldsymbol{\sigma}_{b}\right)+\left(\nabla \mathbf{v}_{b}\right)^{T} \cdot \boldsymbol{\sigma}_{b}+\boldsymbol{\sigma}_{b} \cdot\left(\nabla \mathbf{v}_{b}\right)
$$

In order to enforce incompressibility, the Rayleigh functional can be modified with a Lagrange multiplier term, with a pressure field $P$ acting as the Lagrange multiplier,

$$
\mathcal{R}_{M}=\mathcal{R}-\int d \mathbf{r} P(\mathbf{r}, t) \nabla \cdot\left[\mathbf{v}_{a} \rho_{a}+\mathbf{v}_{b} \rho_{b}\right] .
$$

Taking variational derivatives of $\mathcal{R}_{M}$ with respect to the polymer velocity fields leads to two momentum balance equations:

$$
\begin{aligned}
& \rho_{m} \rho_{a} \frac{\partial \mathbf{v}_{a}}{\partial t}=-\frac{\delta \mathcal{R}_{M}}{\delta \mathbf{v}_{a}}=-\xi\left(\mathbf{v}_{a}-\mathbf{v}_{b}\right)-\nabla \cdot \boldsymbol{\Pi}_{a}-\rho_{a} \nabla P \\
& \rho_{m} \rho_{b} \frac{\partial \mathbf{v}_{b}}{\partial t}=-\frac{\delta \mathcal{R}_{M}}{\delta \mathbf{v}_{b}}=-\xi\left(\mathbf{v}_{b}-\mathbf{v}_{a}\right)-\nabla \cdot \boldsymbol{\Pi}_{b}-\rho_{b} \nabla P
\end{aligned}
$$

where $\rho_{m}$ is the polymer mass per monomer (assumed to be equal for the two types of polymers) and

$$
\begin{aligned}
& \nabla \cdot \boldsymbol{\Pi}_{a}=\nabla \cdot \boldsymbol{\Pi}_{\rho_{a}}+\nabla \cdot \boldsymbol{\Pi}_{\sigma_{a}}, \\
& \nabla \cdot \boldsymbol{\Pi}_{\rho_{a}}=\rho_{a} \nabla \frac{\delta H}{\delta \rho_{a}}, \\
& \nabla \cdot \boldsymbol{\Pi}_{\sigma_{a}}=-\frac{\delta H}{\delta \boldsymbol{\sigma}_{a}}:\left(\nabla \boldsymbol{\sigma}_{a}\right)+\nabla\left(\frac{\delta H}{\delta \boldsymbol{\sigma}_{a}}: \boldsymbol{\sigma}_{a}\right)-2 \nabla \cdot\left(\frac{\delta H}{\delta \boldsymbol{\sigma}_{a}} \cdot \boldsymbol{\sigma}_{a}\right),
\end{aligned}
$$

and similarly for polymer b. For simplicity we assume $\rho_{m}=1, \rho_{a}+\rho_{b}=1$.

Following [5], we define the species velocity difference $\mathbf{w}=\mathbf{v}_{a}-\mathbf{v}_{b}$ and rewrite (30)-(31) in terms of $\mathbf{w}$ and the density averaged velocity $\mathbf{v}=\rho_{a} \mathbf{v}_{a}+\rho_{b} \mathbf{v}_{b}$, obtaining:

$$
\frac{\partial \mathbf{v}}{\partial t}+\mathbf{w} \frac{\partial \rho_{b}}{\partial t}=-\nabla \cdot \boldsymbol{\Pi}_{a}-\nabla \cdot \boldsymbol{\Pi}_{b}-\nabla P
$$




$$
\frac{\partial \mathbf{w}}{\partial t}=-\left(\frac{\xi}{\rho_{a}}+\frac{\xi}{\rho_{b}}\right) \mathbf{w}-\frac{1}{\rho_{a}} \nabla \cdot \boldsymbol{\Pi}_{a}+\frac{1}{\rho_{b}} \nabla \cdot \boldsymbol{\Pi}_{b} .
$$

Often it is the case that the second term on the left hand side of the first equation and the left hand side of the second equation are dominated by the other terms [5]. When these terms are neglected we obtain:

$$
\begin{aligned}
& \frac{\partial \mathbf{v}}{\partial t}=-\nabla \cdot \boldsymbol{\Pi}_{a}-\nabla \cdot \boldsymbol{\Pi}_{b}-\nabla P \\
& \mathbf{w}=-\frac{1}{\xi}\left(\rho_{b} \nabla \cdot \boldsymbol{\Pi}_{a}-\rho_{a} \nabla \cdot \boldsymbol{\Pi}_{b}\right)
\end{aligned}
$$

where incompressibility is enforced by $\nabla \cdot \mathbf{v}=0$. The definitions of $\mathbf{v}$ and $\mathbf{w}$ result in the following equations for the species velocities:

$$
\mathbf{v}_{a}=\mathbf{v}-\frac{\rho_{b}}{\xi} \nabla \cdot \boldsymbol{\Pi}_{a}+\frac{\rho_{a} \rho_{b}}{\xi}\left(\nabla \cdot \boldsymbol{\Pi}_{a}+\nabla \cdot \boldsymbol{\Pi}_{b}\right)
$$

and

$$
\mathbf{v}_{b}=\mathbf{v}-\frac{\rho_{a}}{\xi} \nabla \cdot \boldsymbol{\Pi}_{b}+\frac{\rho_{a} \rho_{b}}{\xi}\left(\nabla \cdot \boldsymbol{\Pi}_{a}+\nabla \cdot \boldsymbol{\Pi}_{b}\right) .
$$

Lastly, Equations (27) and (28) for the stress must be modified in order to restore diagonal dissipative couplings on the components of the stress, corresponding to stress relaxation in a homogeneous polymer melt. We thus make the phenomenological modification, as is done in [7], of adding the terms $-\left(\rho_{a} / \tau_{a}\right) \delta H / \delta \boldsymbol{\sigma}_{a}$ and $-\left(\rho_{b} / \tau_{b}\right) \delta H / \delta \boldsymbol{\sigma}_{b}$ to the right hand side, where $\tau_{a}$ and $\tau_{b}$ are stress relaxation time parameters.

\subsection{Summary}

In summary, our model for an incompressible melt of elastic dumbbells is given by:

$$
\begin{aligned}
\frac{\partial \rho_{a}}{\partial t} & =-\nabla \cdot\left(\mathbf{v}_{a} \rho_{a}\right) \\
\frac{\partial \rho_{b}}{\partial t} & =-\nabla \cdot\left(\mathbf{v}_{b} \rho_{b}\right), \\
\frac{\partial \boldsymbol{\sigma}_{a}}{\partial t} & =-\nabla \cdot\left(\mathbf{v}_{a} \boldsymbol{\sigma}_{a}\right)+\left(\nabla \mathbf{v}_{a}\right)^{T} \cdot \boldsymbol{\sigma}_{a}+\boldsymbol{\sigma}_{a} \cdot\left(\nabla \mathbf{v}_{a}\right)-\frac{\rho_{a}}{\tau_{a}} \frac{\delta H}{\delta \boldsymbol{\sigma}_{a}}
\end{aligned}
$$




$$
\begin{aligned}
\frac{\partial \boldsymbol{\sigma}_{b}}{\partial t} & =-\nabla \cdot\left(\mathbf{v}_{b} \boldsymbol{\sigma}_{b}\right)+\left(\nabla \mathbf{v}_{b}\right)^{T} \cdot \boldsymbol{\sigma}_{b}+\boldsymbol{\sigma}_{b} \cdot\left(\nabla \mathbf{v}_{b}\right)-\frac{\rho_{b}}{\tau_{b}} \frac{\delta H}{\delta \boldsymbol{\sigma}_{b}} \\
\mathbf{v}_{a} & =\mathbf{v}-\frac{\rho_{b}}{\xi} \nabla \cdot \boldsymbol{\Pi}_{a}+\frac{\rho_{a} \rho_{b}}{\xi}\left(\nabla \cdot \boldsymbol{\Pi}_{a}+\nabla \cdot \boldsymbol{\Pi}_{b}\right) \\
\mathbf{v}_{b} & =\mathbf{v}-\frac{\rho_{a}}{\xi} \nabla \cdot \boldsymbol{\Pi}_{b}+\frac{\rho_{a} \rho_{b}}{\xi}\left(\nabla \cdot \boldsymbol{\Pi}_{a}+\nabla \cdot \boldsymbol{\Pi}_{b}\right) \\
\frac{\partial \mathbf{v}}{\partial t} & =-\nabla \cdot \boldsymbol{\Pi}_{a}-\nabla \cdot \boldsymbol{\Pi}_{b}-\nabla P \\
\nabla \cdot \mathbf{v} & =0
\end{aligned}
$$

and

$$
\frac{\delta H}{\delta \mu_{a}}=\frac{\delta H}{\delta \mu_{b}}=0, \frac{\delta H}{\delta \boldsymbol{\epsilon}_{a}}=\frac{\delta H}{\delta \boldsymbol{\epsilon}_{b}}=0,
$$

where

$$
\begin{aligned}
\nabla \cdot \boldsymbol{\Pi}_{a}= & \rho_{a} \nabla \frac{\delta H}{\delta \rho_{a}}-\frac{\delta H}{\delta \boldsymbol{\sigma}_{a}}:\left(\nabla \boldsymbol{\sigma}_{a}\right)+\nabla\left(\frac{\delta H}{\delta \boldsymbol{\sigma}_{a}}: \boldsymbol{\sigma}_{a}\right) \\
& -2 \nabla \cdot\left(\frac{\delta H}{\delta \boldsymbol{\sigma}_{a}} \cdot \boldsymbol{\sigma}_{a}\right), \\
\nabla \cdot \boldsymbol{\Pi}_{b}= & \rho_{b} \nabla \frac{\delta H}{\delta \rho_{b}}-\frac{\delta H}{\delta \boldsymbol{\sigma}_{b}}:\left(\nabla \boldsymbol{\sigma}_{b}\right)+\nabla\left(\frac{\delta H}{\delta \boldsymbol{\sigma}_{b}}: \boldsymbol{\sigma}_{b}\right) \\
& -2 \nabla \cdot\left(\frac{\delta H}{\delta \boldsymbol{\sigma}_{b}} \cdot \boldsymbol{\sigma}_{b}\right) .
\end{aligned}
$$

The general outline for computing the solution goes as follows. Given the fields $\rho_{a}^{n}, \rho_{b}^{n}, \boldsymbol{\sigma}_{a}^{n}, \boldsymbol{\sigma}_{b}^{n}, \mathbf{v}^{n}$ at time $t^{n}$, the first step is to solve for the conjugate fields $\mu_{a}^{n}$, $\mu_{b}^{n}, \boldsymbol{\epsilon}_{a}^{n}, \boldsymbol{\epsilon}_{b}^{n}$ by approximating the solution to the nonlinear optimization problem (49). Next the thermodynamic forces appearing in (50)-(51) are computed in order to update the species velocities $\mathbf{v}_{a}^{n}$ and $\mathbf{v}_{b}^{n}$ in Equations (45)-(46). Lastly, Equations (41)-(44) and Equations (47)-(48) are used to compute the fields $\rho_{a}^{n+1}, \rho_{b}^{n+1}, \boldsymbol{\sigma}_{a}^{n+1}, \boldsymbol{\sigma}_{b}^{n+1}, \mathbf{v}^{n+1}$ at time $t^{n+1}$.

Due to the form of the stress operators $\tilde{\boldsymbol{\sigma}}_{a}$ and $\tilde{\boldsymbol{\sigma}}_{b}$, we have the symmetric properties that $\boldsymbol{\sigma}_{a}=\boldsymbol{\sigma}_{a}^{T}, \boldsymbol{\sigma}_{b}=\boldsymbol{\sigma}_{b}^{T}, \boldsymbol{\epsilon}_{a}=\boldsymbol{\epsilon}_{a}^{T}$, and $\boldsymbol{\epsilon}_{b}=\boldsymbol{\epsilon}_{b}^{T}$. Thus in two dimentions only one of the redundant off diagonal terms needs to be retained for each of these variables in computations. 


\section{$3 \quad$ Numerical Methodology}

In this Section we present a numerical method for Equations (41)-(51) that eliminates the fourth order time-step restrictions associated with explicit methods and allows for the efficient solution of the nonlinear optimization problem given by (49).

We work in two spatial dimensions and use periodic boundary conditions in each direction. The discretization in space is pseudo-spectral, where all derivatives are computed with spectral accuracy for smooth fields using Fast Fourier Transforms. Additionally, after updating all the variables at each timestep, we de-alias $\rho_{a}, \boldsymbol{\sigma}_{a}$, and $\mathbf{v}$ in Fourier space with the smoothing operator $e^{-36\left(|k| /\left|k_{\max }\right|\right)^{36}}$ suggested in [13] as a more accurate alternative to the "twothirds rule".

\subsection{Time Integration Strategy}

The equations of motion for the density and stress variables contain high order derivatives, up to fourth order in $\rho_{a}$ and second order in $\boldsymbol{\sigma}_{a}$. Explicit methods, when applied to such equations, have severe time-step restrictions, requiring prohibitively small time-step sizes. In this section, we present a semi-implicit time-stepping strategy which reduces efficiently the time-step restrictions associated with explicit methods.

Writing Equations (41)-(51) as

$$
\begin{aligned}
& \frac{\partial \rho_{a}}{\partial t}=f_{\rho_{a}}\left(\rho_{a}, \mathbf{v}_{a}\right) \\
& \frac{\partial \boldsymbol{\sigma}_{a}}{\partial t}=f_{\sigma_{a}}\left(\rho_{a}, \boldsymbol{\sigma}_{a}, \boldsymbol{\epsilon}_{a}, \mathbf{v}_{a}\right) \\
& \frac{\partial \mathbf{v}}{\partial t}=f_{v}\left(\rho_{a}, \rho_{b}, \boldsymbol{\sigma}_{a}, \boldsymbol{\sigma}_{b}, \mu_{a}, \mu_{b}, \boldsymbol{\epsilon}_{a}, \boldsymbol{\epsilon}_{b}\right)
\end{aligned}
$$

(with similar equations for polymer b), our approach, similar to [1,3], is to extract a linear operator $L$ from $f$ that contains the linearized, high order derivative terms of $f$ that produce numerical stiffness. We then treat $L$ semiimplicitly, obtaining a time-stepping scheme of the form:

$$
\begin{aligned}
& \frac{\rho_{a}^{n+1}-\rho_{a}^{n}}{\Delta t}=L_{\rho_{a}}^{n+1}+f_{\rho_{a}}^{n}-L_{\rho_{a}}^{n}, \\
& \frac{\boldsymbol{\sigma}_{a}^{n+1}-\boldsymbol{\sigma}_{a}^{n}}{\Delta t}=L_{\sigma_{a}}^{n+1}+f_{\sigma_{a}}^{n}-L_{\sigma_{a}}^{n},
\end{aligned}
$$


and

$$
\frac{\mathbf{v}^{n+1}-\mathbf{v}^{n}}{\Delta t}=L_{v}^{n+1}+f_{v}^{n}-L_{v}^{n}
$$

The first step is to approximate the fields $\mu_{a}$ and $\boldsymbol{\epsilon}_{a}$ to leading order in $\rho_{a}$ and $\boldsymbol{\sigma}_{a}$. From Equation (20) we have that

$$
\mu_{a}=-\log \left(\rho_{a}\right)+\log \left(\frac{2 n_{a}}{Q_{a} Q_{a 0}} \int d \mathbf{r}^{\prime} e^{-\mu_{a}\left(r^{\prime}\right)-\frac{1}{4 R g_{a}^{2}}\left(r-r^{\prime}\right)^{2}:\left[I+\epsilon_{a}(r)+\epsilon_{a}\left(r^{\prime}\right)\right]}\right) .
$$

When the right side is expanded to first order in $\mu_{a}$ and $\boldsymbol{\epsilon}_{a}$, the first term is dominant at high frequencies and therefore we have for large $k$ the relation,

$$
F\left[\mu_{a}\right](k) \approx-F\left[\log \left(\rho_{a}\right)\right](k),
$$

where $F$ denotes the Fourier transform. Expanding the operators $\tilde{\rho}_{a}$ and $\tilde{\boldsymbol{\sigma}}_{a}$ in Equations (20) and (21) to first order in $\mu_{a}$ and $\boldsymbol{\epsilon}_{a}$, we have:

$$
\begin{aligned}
\rho_{a}(\mathbf{r}) \approx & \rho_{a 0}\left(1-\mu_{a}(\mathbf{r})\right)-\frac{\rho_{a 0}}{G} \int d \mathbf{r}^{\prime}\left(g\left(\mathbf{r}, \mathbf{r}^{\prime}\right) \cdot \mu_{a}\left(\mathbf{r}^{\prime}\right)\right) \\
& -\frac{\rho_{a 0}}{4 G R g_{a}^{2}} \int d \mathbf{r}^{\prime}\left(g\left(\mathbf{r}, \mathbf{r}^{\prime}\right) \cdot\left[\left(\mathbf{r}-\mathbf{r}^{\prime}\right)^{2}: \boldsymbol{\epsilon}_{a}\left(\mathbf{r}^{\prime}\right)\right]\right) \\
& -\frac{\rho_{a 0}}{4 G R g_{a}^{2}} \boldsymbol{\epsilon}_{a}(\mathbf{r}): \int d \mathbf{r}^{\prime}\left(g\left(\mathbf{r}, \mathbf{r}^{\prime}\right) \cdot\left(\mathbf{r}-\mathbf{r}^{\prime}\right)^{2}\right)
\end{aligned}
$$

and

$$
\begin{aligned}
\boldsymbol{\sigma}_{a}(\mathbf{r}) \approx & \frac{\rho_{a 0}}{4 G R g_{a}^{2}}\left(1-\mu_{a}(\mathbf{r})\right) \cdot \int d \mathbf{r}^{\prime}\left(g\left(\mathbf{r}, \mathbf{r}^{\prime}\right) \cdot\left(\mathbf{r}-\mathbf{r}^{\prime}\right)^{2}\right) \\
& -\frac{\rho_{a 0}}{4 G R g_{a}^{2}} \int d \mathbf{r}^{\prime}\left(g\left(\mathbf{r}, \mathbf{r}^{\prime}\right) \cdot \mu_{a}\left(\mathbf{r}^{\prime}\right) \cdot\left(\mathbf{r}-\mathbf{r}^{\prime}\right)^{2}\right) \\
& -\frac{\rho_{a 0}}{16 G R g_{a}^{4}} \int d \mathbf{r}^{\prime}\left(g\left(\mathbf{r}, \mathbf{r}^{\prime}\right) \cdot\left(\mathbf{r}-\mathbf{r}^{\prime}\right)^{2} \cdot\left[\left(\mathbf{r}-\mathbf{r}^{\prime}\right)^{2}: \boldsymbol{\epsilon}_{a}\left(\mathbf{r}^{\prime}\right)\right]\right) \\
& -\frac{\rho_{a 0}}{16 G R g_{a}^{4}} \int d \mathbf{r}^{\prime}\left(g\left(\mathbf{r}, \mathbf{r}^{\prime}\right) \cdot\left(\mathbf{r}-\mathbf{r}^{\prime}\right)^{2} \cdot\left[\left(\mathbf{r}-\mathbf{r}^{\prime}\right)^{2}: \boldsymbol{\epsilon}_{a}(\mathbf{r})\right]\right),
\end{aligned}
$$

where

$$
g\left(\mathbf{r}, \mathbf{r}^{\prime}\right)=\exp \left(-\frac{\left|\mathbf{r}-\mathbf{r}^{\prime}\right|^{2}}{4 R g_{a}^{2}}\right), G=\int d \mathbf{r} g\left(\mathbf{r}, \mathbf{r}^{\prime}\right),
$$

and $\rho_{a 0}=2 n_{a} / V$ is the average monomer density of polymer a. The second and third terms in each of the above expansions can be neglected at high 
frequencies (due to the decay of the Gaussian kernel in Fourier space) and after evaluating the remaining Gaussian integrals we are left with:

$$
\begin{aligned}
& \rho_{a}(\mathbf{r}) \approx \rho_{a 0}\left(1-\mu_{a}(\mathbf{r})\right)-\frac{\rho_{a 0}}{2} \epsilon_{11_{a}}(\mathbf{r})-\frac{\rho_{a 0}}{2} \epsilon_{22_{a}}(\mathbf{r}), \\
& \sigma_{11_{a}}(\mathbf{r}) \approx \frac{\rho_{a 0}}{2}\left(1-\mu_{a}(\mathbf{r})\right)-\frac{3 \rho_{a 0}}{4} \epsilon_{11_{a}}(\mathbf{r})-\frac{\rho_{a 0}}{4} \epsilon_{22_{a}}(\mathbf{r}), \\
& \sigma_{12_{a}}(\mathbf{r}) \approx \frac{\rho_{a 0}}{2} \epsilon_{12_{a}}(\mathbf{r}), \\
& \sigma_{22_{a}}(\mathbf{r}) \approx \frac{\rho_{a 0}}{2}\left(1-\mu_{a}(\mathbf{r})\right)-\frac{3 \rho_{a 0}}{4} \epsilon_{22_{a}}(\mathbf{r})-\frac{\rho_{a 0}}{4} \epsilon_{11_{a}}(\mathbf{r}) .
\end{aligned}
$$

Solving for $\boldsymbol{\epsilon}_{a}$, we have the following relations for large $k$ :

$$
\begin{aligned}
& F\left[\epsilon_{11_{a}}\right](k) \approx \frac{2}{\rho_{a 0}} F\left[\frac{1}{2} \rho_{a}-\sigma_{11_{a}}\right](k), \\
& F\left[\epsilon_{22_{a}}\right](k) \approx \frac{2}{\rho_{a 0}} F\left[\frac{1}{2} \rho_{a}-\sigma_{22_{a}}\right](k), \\
& F\left[\epsilon_{12_{a}}\right](k) \approx \frac{2}{\rho_{a 0}} F\left[-\sigma_{12_{a}}\right](k) .
\end{aligned}
$$

Following the methodology in $[1,3]$, we next linearize the operator $f$ about a homogeneous solution, $\overline{\boldsymbol{\sigma}}_{a}=\frac{1}{2} \bar{\rho}_{a} I, \overline{\mathbf{v}}=0, \overline{\boldsymbol{\epsilon}}_{a}=0$, using the relations (59) and (67)-(69). For $\rho_{a}$, we have that

$$
\frac{\partial \rho_{a}}{\partial t}=\nabla \cdot\left(\frac{\rho_{a}^{2} \rho_{b}^{2}}{\xi} \nabla \cdot \frac{\delta H}{\delta \rho_{a}}\right)-\nabla \cdot\left(\frac{\rho_{a}^{2} \rho_{b}^{2}}{\xi} \nabla \cdot \frac{\delta H}{\delta \rho_{b}}\right)+\ldots
$$

and using $\rho_{a}+\rho_{b}=1$ we find that

$$
L_{\rho_{a}}=\frac{\bar{\rho}_{a} \bar{\rho}_{b}}{\bar{\xi}} \nabla^{2} \rho_{a}-\frac{2 \bar{\rho}_{a}^{2} \bar{\rho}_{b}^{2} \chi_{1}}{\bar{\xi}} \nabla^{2} \rho_{a}-\frac{2 \bar{\rho}_{a}^{2} \bar{\rho}_{b}^{2} \chi_{2}}{\bar{\xi}} \nabla^{4} \rho_{a}
$$

For $\boldsymbol{\sigma}_{a}$, with $\Pi_{\sigma_{a} x}$ denoting the $x$ component of $\boldsymbol{\Pi}_{\sigma_{a}}$, we have that

$$
\begin{aligned}
\frac{\partial \sigma_{11_{a}}}{\partial t}= & -\sigma_{11_{a}} \frac{\partial}{\partial x}\left(\frac{\rho_{b}}{\xi} \Pi_{\sigma_{a} x}\right)+\sigma_{11_{a}} \frac{\partial}{\partial y}\left(\frac{\rho_{b}}{\xi} \Pi_{\sigma_{a} y}\right) \\
& -\left(\rho_{a} / \tau_{a}\right) \frac{\delta H}{\delta \sigma_{11_{a}}}+\ldots, \\
\frac{\partial \sigma_{22_{a}}}{\partial t}= & -\sigma_{22_{a}} \frac{\partial}{\partial y}\left(\frac{\rho_{b}}{\xi} \Pi_{\sigma_{a} y}\right)+\sigma_{22_{a}} \frac{\partial}{\partial x}\left(\frac{\rho_{b}}{\xi} \Pi_{\sigma_{a} x}\right)
\end{aligned}
$$




$$
\begin{aligned}
& -\left(\rho_{a} / \tau_{a}\right) \frac{\delta H}{\delta \sigma_{22_{a}}}+\ldots, \\
\frac{\partial \sigma_{12_{a}}}{\partial t}= & -\sigma_{11_{a}} \frac{\partial}{\partial x}\left(\frac{\rho_{b}}{\xi} \Pi_{\sigma_{a} y}\right)-\sigma_{22_{a}} \frac{\partial}{\partial y}\left(\frac{\rho_{b}}{\xi} \Pi_{\sigma_{a} x}\right) \\
& -\left(\rho_{a} / \tau_{a}\right) \frac{\delta H}{\delta \sigma_{12_{a}}}+\ldots,
\end{aligned}
$$

where the last terms on the right hand side are retained in order to reduce the dependence of the time-step restriction on the stress relaxation parameter $\tau_{a}$. Since we are linearizing about $\overline{\boldsymbol{\epsilon}}_{a}=0$, many of the high order terms from $\boldsymbol{\Pi}_{\sigma_{a}}$ vanish and we are left with

$$
\begin{aligned}
L_{\sigma_{11_{a}}} & =\frac{\bar{\rho}_{a} \bar{\rho}_{b}}{2 \bar{\xi}} \nabla^{2} \sigma_{11_{a}}-\frac{2}{\tau_{a}} \sigma_{11_{a}}, \\
L_{\sigma_{22_{a}}} & =\frac{\bar{\rho}_{a} \bar{\rho}_{b}}{2 \bar{\xi}} \nabla^{2} \sigma_{22_{a}}-\frac{2}{\tau_{a}} \sigma_{22_{a}}, \\
L_{\sigma_{12_{a}}} & =\frac{\bar{\rho}_{a} \bar{\rho}_{b}}{\bar{\xi}} \nabla^{2} \sigma_{12_{a}}-\frac{2}{\tau_{a}} \sigma_{12_{a}},
\end{aligned}
$$

where we have retained only the second order diagonal terms and the terms involving $\tau_{a}$. For the velocity $\mathbf{v}$ we note that $f_{v}$ does not depend on the velocity and therefore we set $L_{v}=f_{v}$.

So far we have neglected the high order $\rho_{a}$ terms appearing in the evolution equation for the stress variable. In order to remove the stiffness associated with these terms, we treat $\rho_{a}$ implicitly in the right hand side of the equation for the stress. In summary, our numerical integration scheme is given by:

$$
\begin{aligned}
\frac{\rho_{a}^{n+1}-\rho_{a}^{n}}{\Delta t}= & -C_{1} \nabla^{4} \rho_{a}^{n+1}+C_{2} \nabla^{2} \rho_{a}^{n+1}+f_{\rho_{a}}\left(\rho_{a}^{n}, \mathbf{v}_{a}^{n}\right) \\
& +C_{1} \nabla^{4} \rho_{a}^{n}-C_{2} \nabla^{2} \rho_{a}^{n} \\
\frac{\sigma_{i j_{a}}^{n+1}-\sigma_{i j_{a}}^{n}=}{\Delta t}{ }^{i j} \nabla^{2} \sigma_{i j_{a}}^{n+1}-C_{4} \sigma_{i j_{a}}^{n+1}+f_{\sigma_{a}}\left(\rho_{a}^{n+1}, \boldsymbol{\sigma}_{a}^{n}, \boldsymbol{\epsilon}_{a}^{n}, \mathbf{v}_{a}^{*}\right) & -C_{3}^{i j} \nabla^{2} \sigma_{i j_{a}}^{n}+C_{4} \sigma_{i j_{a}}^{n}, \\
\frac{\mathbf{v}^{n+1}-\mathbf{v}^{n}}{\Delta t}= & f_{v}\left(\rho_{a}^{n+1}, \rho_{b}^{n+1}, \boldsymbol{\sigma}_{a}^{n+1}, \boldsymbol{\sigma}_{b}^{n+1}, \mu_{a}^{n+1}, \mu_{b}^{n+1}, \boldsymbol{\epsilon}_{a}^{n+1}, \boldsymbol{\epsilon}_{b}^{n+1}\right),
\end{aligned}
$$


where

$$
\mathbf{v}_{a}^{*}=\mathbf{v}_{a}\left[\mathbf{v}^{n}, \rho_{a}^{n+1}, \rho_{b}^{n+1}, \boldsymbol{\sigma}_{a}^{n}, \boldsymbol{\sigma}_{b}^{n}, \mu_{a}^{n}, \mu_{b}^{n}, \boldsymbol{\epsilon}_{a}^{n}, \boldsymbol{\epsilon}_{b}^{n}\right] .
$$

As in $[1,3]$, the constants are given by the maximum value of the coefficients of the linearized terms,

$$
\begin{aligned}
& C_{1}=\max _{r}\left[\frac{\rho_{a}(\mathbf{r}) \rho_{b}(\mathbf{r})}{\xi(\mathbf{r})}\left(1-2 \rho_{a}(\mathbf{r}) \rho_{b}(\mathbf{r}) \chi_{1}\right)\right], C_{2}=\max _{r}\left[\frac{2 \rho_{a}(\mathbf{r})^{2} \rho_{b}(\mathbf{r})^{2} \chi_{2}}{\xi(\mathbf{r})}\right] \\
& 2 C_{3}^{11}=2 C_{3}^{22}=C_{3}^{12}=\max _{r}\left[\frac{\rho_{a}(\mathbf{r}) \rho_{b}(\mathbf{r})}{\xi(\mathbf{r})}\right], C_{4}=\frac{2}{\tau_{a}}
\end{aligned}
$$

which can either be calculated analytically or computed at each time-step.

\subsection{Mean-field Computation}

Since the mean-field approximation is employed to determine the chemical potential and elastic strain fields, the nonlinear optimization problem,

$$
\frac{\delta H}{\delta \mu_{a}}\left[\rho_{a}^{n}, \mu_{a}^{n}, \boldsymbol{\epsilon}_{a}^{n}\right]=0, \frac{\delta H}{\delta \boldsymbol{\epsilon}_{a}}\left[\boldsymbol{\sigma}_{a}^{n}, \mu_{a}^{n}, \boldsymbol{\epsilon}_{a}^{n}\right]=0,
$$

must be solved at each time-step $t_{n}$ (and similarly for polymer b). Following [2], we use a gradient flow approach, writing (83) as

$$
\frac{\partial \mu_{a}}{\partial s}=\frac{\delta H}{\delta \mu_{a}}\left[\rho_{a}^{n}, \mu_{a}, \boldsymbol{\epsilon}_{a}\right]
$$

and

$$
\frac{\partial \boldsymbol{\epsilon}_{a}}{\partial s}=\frac{\delta H}{\delta \boldsymbol{\epsilon}_{a}}\left[\boldsymbol{\sigma}_{a}^{n}, \mu_{a}, \boldsymbol{\epsilon}_{a}\right]
$$

where $s$ is a fictitious time variable and the steady state solution corresponds to the mean-fields $\mu_{a}^{n}$ and $\boldsymbol{\epsilon}_{a}^{n}$. We then discretize Equations (84) and (85), following [2] using the two-step Seidel type iteration scheme

$$
\frac{\mu_{a}^{i+1, n}-\mu_{a}^{i, n}}{\Delta s}=\frac{\delta H}{\delta \mu_{a}}\left[\rho_{a}^{n}, \mu_{a}^{i, n}, \boldsymbol{\epsilon}_{a}^{i, n}\right],
$$




$$
\frac{\boldsymbol{\epsilon}_{a}^{i+1, n}-\boldsymbol{\epsilon}_{a}^{i, n}}{\Delta s}=\frac{\delta H}{\delta \boldsymbol{\epsilon}_{a}}\left[\boldsymbol{\sigma}_{a}^{n}, \mu_{a}^{i+1, n}, \boldsymbol{\epsilon}_{a}^{i, n}\right]
$$

and relax until

$$
\max _{k, l=1,2}\left(\left\|\mu_{a}^{i+1, n}-\mu_{a}^{i, n}\right\|_{2},\left\|\epsilon_{k l_{a}}^{i+1, n}-\epsilon_{k l_{a}}^{i, n}\right\|_{2}\right) \leq \text { tol }
$$

Though this iteration scheme costs twice as much per iteration as the onestep forward Euler scheme, we find that it is more robust, converging for nonsmooth chemical potential and elastic strain fields when the Euler method fails to converge.

Because the functionals in (83) are invariant under constant shifts in $\mu_{a}$, we enforce

$$
\int d \mathbf{r} \mu_{a}^{i, n}(\mathbf{r})=0
$$

after updating $\mu_{a}^{i, n}$ at each pseudo time-step $\Delta s$.

With an arbitrary initial guess $\mu_{a}^{0, n}$ and $\boldsymbol{\epsilon}_{a}^{0, n}$, the relaxation scheme given by (86) and (87) may require thousands of iterations in order to converge within a desired tolerance. Furthermore, at each pseudo time-step $s_{i}$ the two dimensional integrals over $\mathbb{R}^{2}$ appearing in Equations (20) and (21) must be computed at each point in the physical domain. Thus with a spatial resolution of $N \times N$ points, the computational cost of just one pseudo time-step is $O\left(N^{4}\right)$.

In order to bring down the computational cost, we first improve upon the initial guess. Since we have solved the optimization problem at times $t_{n-1}$ and time $t_{n-2}$, we can use the mean-fields at these times to construct an extrapolated initial guess:

$$
\mu_{a}^{0, n}=2 \mu_{a}^{n-1}-\mu_{a}^{n-2}, \quad \boldsymbol{\epsilon}_{a}^{0, n}=2 \boldsymbol{\epsilon}_{a}^{n-1}-\boldsymbol{\epsilon}_{a}^{n-2} .
$$

With such a procedure the number of iterations required for convergence is reduced to $O(10)$ when the time-step $\Delta t$ is not too large compared to the magnitude of the fluid velocity (for larger time-steps extrapolation provides a less accurate initial guess and the relaxation scheme then requires more iterations in order to converge).

To bring down the computational cost associated with the integration required at each pseudo time-step, we first observe that the integrands appearing in (20) and (21) decay exponentially. This implies that the error caused by integrating 
over a finite region decreases exponentially as the domain of integration increases. Thus the integrals in (20) and (21) can be restricted to a small region centered about $\mathbf{r}$ while maintaining accuracy. Additionally, we find that the spatial resolution used in the integration can be reduced while still accurately resolving the operators $\tilde{\rho}_{a}(\mathbf{r})$ and $\tilde{\boldsymbol{\sigma}}_{a}(\mathbf{r})$.

As an example, we numerically approximate the integral of the function

$$
e^{-\left(x^{2}+y^{2}\right) / 4} \cdot x^{2}[1+\tanh (5 \cos (8 \pi x / 40) \cos (8 \pi y / 40))]
$$

similar in form to the integrands in (20) and (21), over $\mathbb{R}^{2}$ using several spatial resolutions and domain sizes. In Table 1, we observe that the error created by reducing the size of the domain of integration from $40 \times 40$ to $20 \times 20$ and increasing the grid spacing by a factor of 2 is 0.033 . Furthermore, the integrals in (20) and (21) are normalized to obtain $\tilde{\rho}_{a}(\mathbf{r})$ and $\tilde{\boldsymbol{\sigma}}_{a}(\mathbf{r})$, which are $\mathrm{O}(1)$, and thus the error contribution from the integration to the mean-field approximation will be even smaller, $O\left(10^{-3}\right)$, in the context of the optimization problem. We note that on a domain of size $40 \times 40$ using a $128 \times 128$ grid, a second order finite difference approximation to the system of PDEs in the model creates an error on the order of $40^{2} / 128^{2}=0.098$ that is comparable to the error contributed from the integration under the $L^{2}$-norm.

Table 1

Integration resolution comparison

\begin{tabular}{c|c|c}
\hline domain of integration & integration resolution & value of integral \\
\hline $20 \times 20$ & $32 \times 32$ & 29.92850069 \\
\hline $20 \times 20$ & $64 \times 64$ & 29.96182141 \\
\hline $40 \times 40$ & $128 \times 128$ & 29.96182142
\end{tabular}

Even with the savings gained by reducing the resolution and domain of integration, the operators must still be evaluated at every mesh point $\mathbf{r}$ in the physical domain. To bring the simulation time down further, we divide the physical domain into 32 equally sized pieces and evaluate the operators in parallel at each pseudo time-step (the number of processors was chosen in order to balance the cost of mathematical operations and communication between processors). The computational savings of our approach are illustrated in Table 2. 
Table 2

CPU time per pseudo time-step $\Delta s$ of the nonlinear optimization problem (averaged over a sample size of 5)

\begin{tabular}{c|c|c|c}
\hline domain of integration & integration resolution & \# of processors & CPU time (sec) \\
\hline $20 \times 20$ & $32 \times 32$ & 32 & 1.3 \\
\hline $40 \times 40$ & $128 \times 128$ & 32 & 14.7 \\
\hline $40 \times 40$ & $128 \times 128$ & 1 & 396.2
\end{tabular}

\section{Numerical Results}

In this section we illustrate the effectiveness of the numerical method with several examples. In Section 4.1 we estimate the time-step restriction of the semi-implicit method presented in Section 3.1. In Section 4.2 we provide simulation results for a phase separating, symmetric melt in an initially quiescent flow. Finally, in Section 4.3 we investigate the effects of asymmetry between the fluid components. All simulations in this work were carried out on 32 P655 nodes on Datastar at the San Diego Supercomputer Center.

\subsection{Time-step restriction of semi-implicit integration scheme}

We consider a domain of size $[40 R g, 40 R g]$ and use the initial conditions $\rho_{a}^{0}(x, y)=0.5+0.1 \cos (8 \pi x / 40 R g) \cos (8 \pi y / 40 R g), \rho_{b}^{0}=1-\rho_{a}^{0}, \boldsymbol{\epsilon}_{a}^{0}=\boldsymbol{\epsilon}_{b}^{0}=0$ (which determines $\boldsymbol{\sigma}_{a}^{0}$ and $\boldsymbol{\sigma}_{b}^{0}$ through Equation (83)), and $\mathbf{v}^{0}=0$ and the parameters $\chi_{1}=2, \chi_{2}=0.5, R g_{a}=R g_{b}=1, \xi_{a 0}=\xi_{b 0}=1$, and $\tau_{a}=\tau_{b}=0.2$. For the constants in the semi-implicit integration, we use $C_{1}=1, C_{2}=0.25$, $C_{3}^{12}=2, C_{3}^{i i}=1$, and $C_{4}=20$. For the nonlinear optimization problem, we set $\Delta s=0.9$, tol $=10^{-3}$, and compute all integrals over a region of size $[20 R g \times 20 R g]$. For the spatial resolutions $32 \times 32,64 \times 64$ and $128 \times 128$ we compute the integrals (20)-(21) using the resolutions $16 \times 16,32 \times 32$ and $32 \times 32$.

In Table 3, we list the maximum time-step size $\Delta t$ for which the semi-implicit integration scheme can complete 200 time-steps without diverging (we say that the scheme diverges if the density or diagonal components of the stress go below zero). Here we observe a linear decrease in the size of the maximum allowable time-step as the spatial resolution is increased, which is due to the first order derivative terms appearing in Equations (41)-(51). In contrast, due to the fourth order derivative terms appearing in the equations for the density, explicit methods exhibit a quartic decrease in the size of the maximum allowable time-step. 
Table 3

Resolution vs. maximum time-step

\begin{tabular}{c|c|c|c}
\hline resolution & $32 \times 32$ & $64 \times 64$ & $128 \times 128$ \\
\hline $\max \Delta t$ & 10.4 & 6.4 & 4.6
\end{tabular}

4.2 Phase separation of a symmetric melt of elastic dumbbells in an initially quiescent flow

In this example we use the random initial conditions $\rho_{a}^{0}=0.5+0.1 U[-1,1]$ (where $U$ is a uniform random variable), $\rho_{b}^{0}=1-\rho_{a}^{0}, \boldsymbol{\epsilon}_{a}^{0}=\boldsymbol{\epsilon}_{b}^{0}=0$, and $\mathbf{v}^{0}=0$ and the parameters $\chi_{1}=2, \chi_{2}=0.05, R g_{a}=R g_{b}=1, \xi_{a 0}=\xi_{b 0}=1$, and $\tau_{a}=\tau_{b}=0.1$. For the constants in the semi-implicit integration, we use $C_{1}=1, C_{2}=0.025, C_{3}^{12}=2, C_{3}^{i i}=1$, and $C_{4}=40$. We discretize the domain $[0,40 R g] \times[0,40 R g]$ with a grid of size $128 \times 128$ points and for the nonlinear optimization problem we use the pseudo time-step $\Delta s=0.9$, tolerance tol $=10^{-3}$, and evaluate the integrals (20)-(21) over the domain $[0,20 R g] \times[0,20 R g]$ using a resolution of $32 \times 32$ gridpoints

We integrate up to time $t=50$ using the time-step $\Delta t=0.05$ and observe the classic pattern of symmetric phase separation, with polymer a-rich phases and polymer b-rich phases separated by a thin transition layer (Figure 1). In the contour plots of the components of the stress, we observe less stress in vertically aligned regions and higher stress in horizontally aligned regions, as the fluid deforms in order to align the polymers and ultimately minimize the free energy of the system.

For the above parameter values we find that the average number of iterations per time-step required to solve the nonlinear optimization problem is 15.97 and the total runtime is 11 hours and 22 minutes. We note that the forward Euler method fails to converge for time-steps as small as $\Delta t=3 \cdot 10^{-3}$ (using the above parameters) and thus the runtime associated with an explicit method will be on the order of weeks for similar parameter values. Without the savings gained by efficiently solving the nonlinear optimization problem, the runtime will be even longer, on the order of months.

\subsection{Effects of asymmetry between the fluid components}

\subsubsection{Droplet deformation}

Here we investigate the effects of asymmetry in the polymer stress and friction parameters starting with an elliptical droplet. We use the initial conditions 

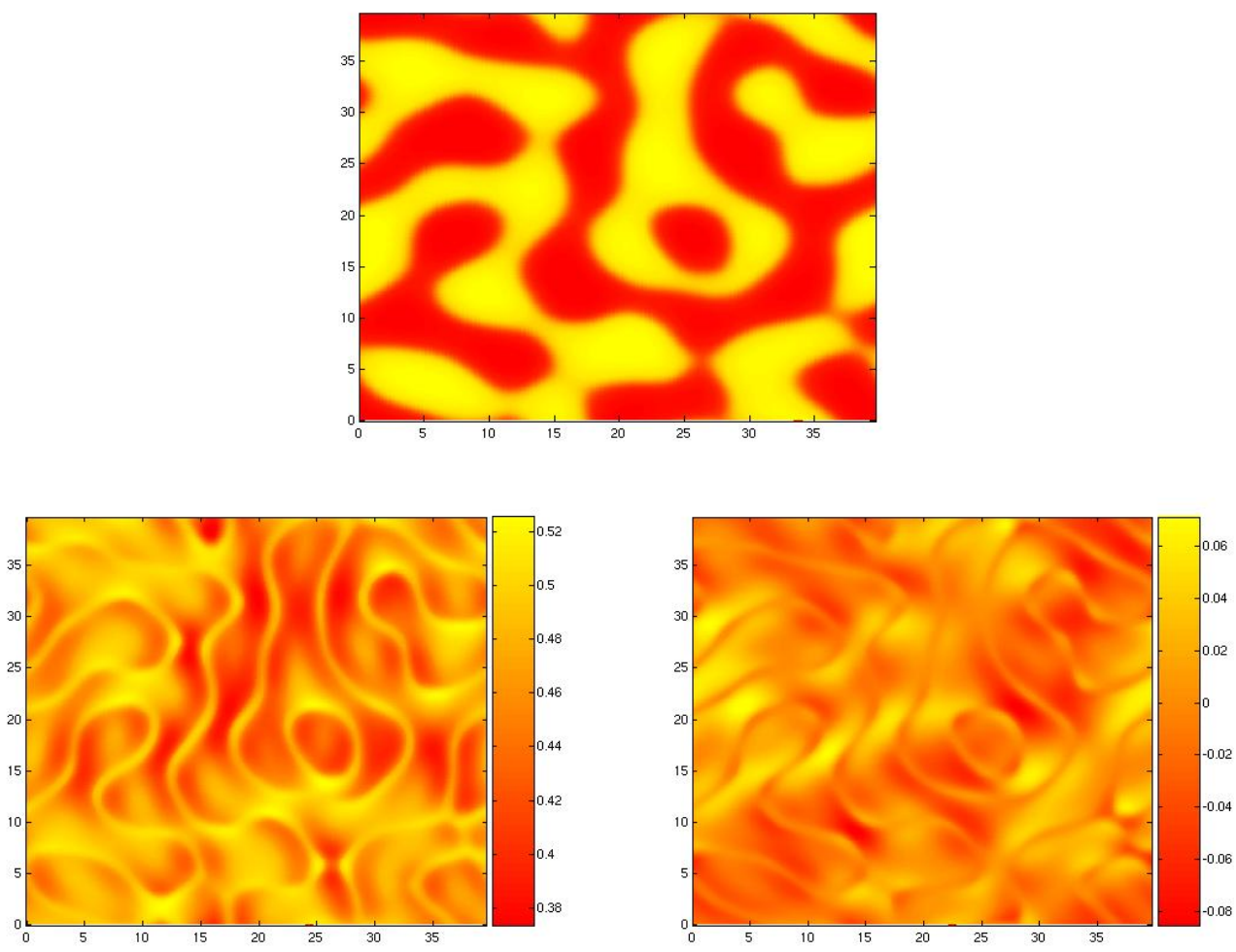

Fig. 1. Contour plots at time $t=50$ of $\rho_{a}$ (top, yellow corresponds to $a$ rich regions), $\sigma_{11_{a}}+\sigma_{11_{b}}$ (bottom left), and $\sigma_{12_{a}}+\sigma_{12_{b}}$ (bottom right). In the bottom left plot, yellow corresponds to higher (bulk) stress and is located in the horizontally aligned regions and the fluid interfaces, whereas red corresponds to lower stress and is located in the vertically aligned regions. In the bottom right, yellow and red correspond to higher (shear) stress, located in the horizontally aligned regions, and orange corresponds to lower stress, located in the vertically aligned regions.

(plotted in Figure 2),

$$
\rho_{a}^{0}=0.5+0.45 \cdot \tanh \left(\sqrt{3 \cdot(x-20)^{2}+(y-20)^{2}}-9\right)
$$

$\rho_{b}^{0}=1-\rho_{a}^{0}, \boldsymbol{\epsilon}_{a}^{0}=\boldsymbol{\epsilon}_{b}^{0}=0$, and $\mathbf{v}^{0}=0$ and the parameters $\chi_{1}=1.5, \chi_{2}=0.25$, and $R g_{a}=R g_{b}=1$. In the first example we use $\xi_{a 0}=10, \xi_{b 0}=0.1$ and $\tau_{a}=10, \tau_{b}=0.1$ and in the second example we reverse the parameters, setting $\xi_{a 0}=0.1, \xi_{b 0}=10$ and $\tau_{a}=0.1, \tau_{b}=10$. For the constants in the semi-implicit integration, we use $C_{1}=10, C_{2}=1.25, C_{3}^{12}=20, C_{3}^{i i}=10$, and $C_{4}^{a}=0.4, C_{4}^{b}=40$ in the first example and $C_{4}^{a}=40, C_{4}^{b}=0.4$ in the second example. We discretize the domain $[0,40 R g] \times[0,40 R g]$ with a grid of size $64 \times 64$ points and for the nonlinear optimization problem we use the pseudo time-step $\Delta s=0.9$, tolerance $t o l=10^{-3}$, and evaluate the integrals (20)-(21) over the domain $[0,20 R g] \times[0,20 R g]$ using a resolution of $32 \times 32$ gridpoints. For both examples we use the time-step $\Delta t=0.01$ up to time $t=0.25$ (in 
order to satisfy the CFL condition) and $\Delta t=0.25$ up to time $t=150$.

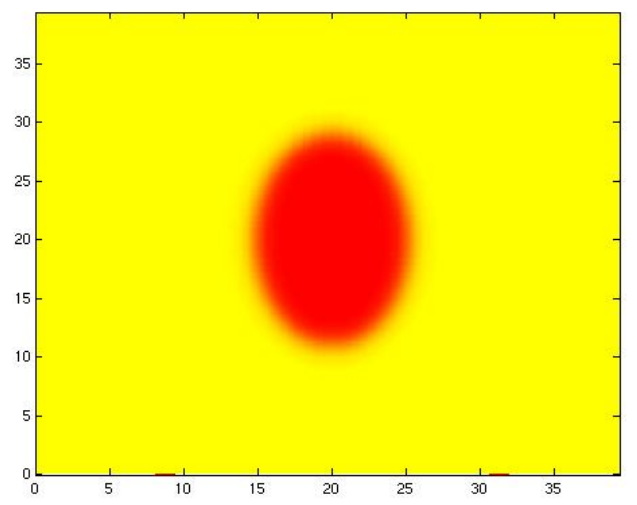

Fig. 2. Initial profile of the droplet given by Equation (91). Yellow corresponds to the a-rich region. The initial area of the droplet is $146 R g^{2}$.

In Figure 3 we plot the droplets for each of the two examples at time $t=150$, as well as the total polymer stresses $\sigma_{11_{a}}+\sigma_{11_{b}}$ and $\sigma_{12_{a}}+\sigma_{12_{b}}$. On the left, the fluid in the droplet has smaller friction and stress relaxation parameters, resulting in the inability of the droplet to store stress (compared to the droplet on the right) and a greater mobility of the fluid in the droplet. Comparing the two examples, we observe that the droplet on the left forms the energy minimizing circular shape more quickly, whereas the droplet on the right, due to its ability to store stress and smaller mobility coefficient, relaxes more slowly. In the plots of the stress, we observe that the droplet on the left has lower bulk stress in the center of the droplet (middle left plot) and shear stress surrounding the fluid interface (lower left plot) in comparison to the droplet on the right.

\subsubsection{Viscoelastic phase separation}

When strong asymmetries exist between the two components of the fluid, it has been observed that phase inversion is possible [18]. Following [19], we modify the frictional terms and replace Equation (23) with

$$
\xi=\frac{\xi_{a 0}-\xi_{b 0}}{2} \cdot \tanh \left(\frac{1}{0.01 \cdot \tan \left(\pi \rho_{a 0}\right)}-\frac{1}{0.01 \cdot \tan \left(\pi \rho_{a}\right)}\right)+\frac{\xi_{a 0}+\xi_{b 0}}{2}
$$

which generates strong asymmetry between the mobilities of the two fluid components. We use the random initial conditions $\rho_{a}^{0}=0.3+0.001 U[-1,1]$ $\left(\rho_{a 0}=0.3\right), \rho_{b}^{0}=1-\rho_{a}^{0}, \boldsymbol{\epsilon}_{a}^{0}=\boldsymbol{\epsilon}_{b}^{0}=0$, and $\mathbf{v}^{0}=0$ and the parameters $\chi_{1}=1.5, \chi_{2}=0.25, R g_{a}=R g_{b}=1, \xi_{a 0}=1, \xi_{b 0}=0.1, \tau_{a}=1, \tau_{b}=0.1$. For the constants in the semi-implicit integration, we use $C_{1}=10, C_{2}=1.25$, 
$C_{3}^{12}=20, C_{3}^{i i}=10, C_{4}^{a}=4$, and $C_{4}^{b}=40$. We discretize the domain $[0,40 R g] \times$ $[0,40 R g]$ with a grid of size $64 \times 64$ points and for the nonlinear optimization problem we use the pseudo time-step $\Delta s=0.9$, tolerance $t o l=10^{-3}$, and evaluate the integrals (20)-(21) over the domain $[0,20 R g] \times[0,20 R g]$ using a resolution of $32 \times 32$ gridpoints.

We use the time-step $\Delta t=0.5$ and in Figure 4 we plot the density at times $t=2900, t=3480$, and $t=4000$. Initially, due to the greater mobility of the polymer $b$ molecules, we observe the nucleation of polymer $b$ molecules into droplets. Since polymer $b$ is the majority phase $\left(\rho_{b 0}=0.7\right)$, the droplets grow in size and a network is then formed by the polymer $a$ molecules. Finally, as the droplets continue to grow, the network breaks down and phase inversion occurs.

\section{Concluding remarks}

We presented a model for an incompressible melt of elastic dumbbell polymers that connects the thermodynamic forces acting on the polymers with the dynamics of the flow via a statistical field theory. We imposed a mean-field approximation and then developed an efficient, semi-implicit numerical method for the resulting system of PDEs, as well as a procedure to expedite the nonlinear optimization component by exploiting the structure of the integrands and by a parallel iterative approach. The semi-implicit method effectively removes the high order stability constraints associated with explicit methods, allowing for much larger time-steps to be taken. Additionally, the computational cost associated with the nonlinear optimization problem was reduced by several orders of magnitude, allowing simulations that would normally take months to be completed in just a few hours. We believe that these computational advances will pave the way for studies of the coupled flow and microstructure of more realistic multi-bead models of inhomogeneous polymeric fluids.

\section{Acknowledgments}

This work was supported in part by NSF grants DMG0609996 and DMR0603710, NSF IGERT grant DGE02-21715 and in part by NSF through the San Diego Supercomputer Center under grant DMR080001N using Datastar. The authors would like to thank Tanya Chantawansri for her contributions to the parallel implementation of the numerical method presented in this paper. 


\section{References}

[1] V. E. Badalassi, H. D. Ceniceros, S. Banerjee, Computation of Multiphase Systems with Phase Field Models, J. Comput. Phys., 190 (2003), pp. 371-397.

[2] H. D. Ceniceros and G. H. Fredrickson, Numerical solution of polymer selfconsistent field theory, Multiscale Modeling and Simulation, 2 (3) (2004), pp. $452-474$.

[3] H. D. Ceniceros and G. O. Mohler, A practical splitting method for stiff SDEs with applications to problems with small noise, Multiscale Modeling and Simulation, 6 (1) (2007), pp. 212-227.

[4] M. Doi and S. F. Edwards, The Theory of Polymer Dynamics, Oxford Press, 1986.

[5] M. Doi and A. Onuki, Dynamic coupling between stress and composition in polymer solutions and blends, J. Phys. II 2 (1992), pp. 1631-1656.

[6] J. Fraaije, B. A. C. Van Vlimmeren, N. M. Maurits, M. Postma, O. A. Evers, C. Hoffmann, P. Altevogt, and G. Goldbeck-Wood, The dynamic mean-field density functional method and its application to the mesoscopic dynamics of quenched block copolymer melts, J. Chem. Phys., 106 (1997), pp. 4260-4269.

[7] G. H. Fredrickson, Dynamics and rheology of inhomogeneous polymeric fluids: A complex Langevin approach, J. Chem. Phys. 117 (14) (2002), pp. 6810-6820.

[8] G. H. Fredrickson, The Equilibrium Theory of Inhomogeneous Polymers, Oxford Press, 2006.

[9] Hall, D. M. Lookman, T. Fredrickson, G. H. Banerjee, S., Numerical method for hydrodynamic transport of inhomogeneous polymer melts J. Comput. Phys., 224 (2007), pp. 681-698.

[10] E. Helfand, Theory of inhomogeneous polymers: Fundamentals of the Gaussian random-walk model, J. Chem. Phys., 62 (1975), pp. 999-1005.

[11] P. C. Hohenberg and B. I. Halperin, Theory of dynamic critical phenomena, Reviews of Modern Physics, 49 (1977), pp. 435-479.

[12] K. M. Hong and J. Noolandi, Theory of inhomogeneous multicomponent polymer systems, Macromolecules, 14 (1981), pp. 727-736.

[13] T. Y. Hou and R. Li, Computing nearly singular solutions using pseudo-spectral methods J. Comput. Phys., 226 (2007), pp. 379-397.

[14] L. Leibler, The theory of microphase separation in block copolymers, Macromolecules, 13 (1980), pp. 1602-1617.

[15] M. W. Matsen, The standard Gaussian model for block copolymer melts, J. Physics-Condensed Matter, 14 (2002), pp. R21-R47. 
[16] M. W. Matsen and M. Schick, Self-assemply of block copolymers, Current Opinion in Colloid and Interface Science, 1 (1996), pp. 329-336.

[17] S. T. Milner, Dynamical theory of concentration fluctuations in polymer solutions under shear, Phys. Rev. E 48 (1993), pp. 3674-3691.

[18] H. Tanaka and T. Araki, Phase inversion during viscoelastic phase separation: roles of bulk and shear relaxation moduli, Phys. Rev. Lett. 78 (26) (1997), pp. 4966-4969.

[19] D. Zhou, P. Zhang, and W. E, Modified models of polymer phase separation, Phys. Rev. E 73, 061801 (2006). 

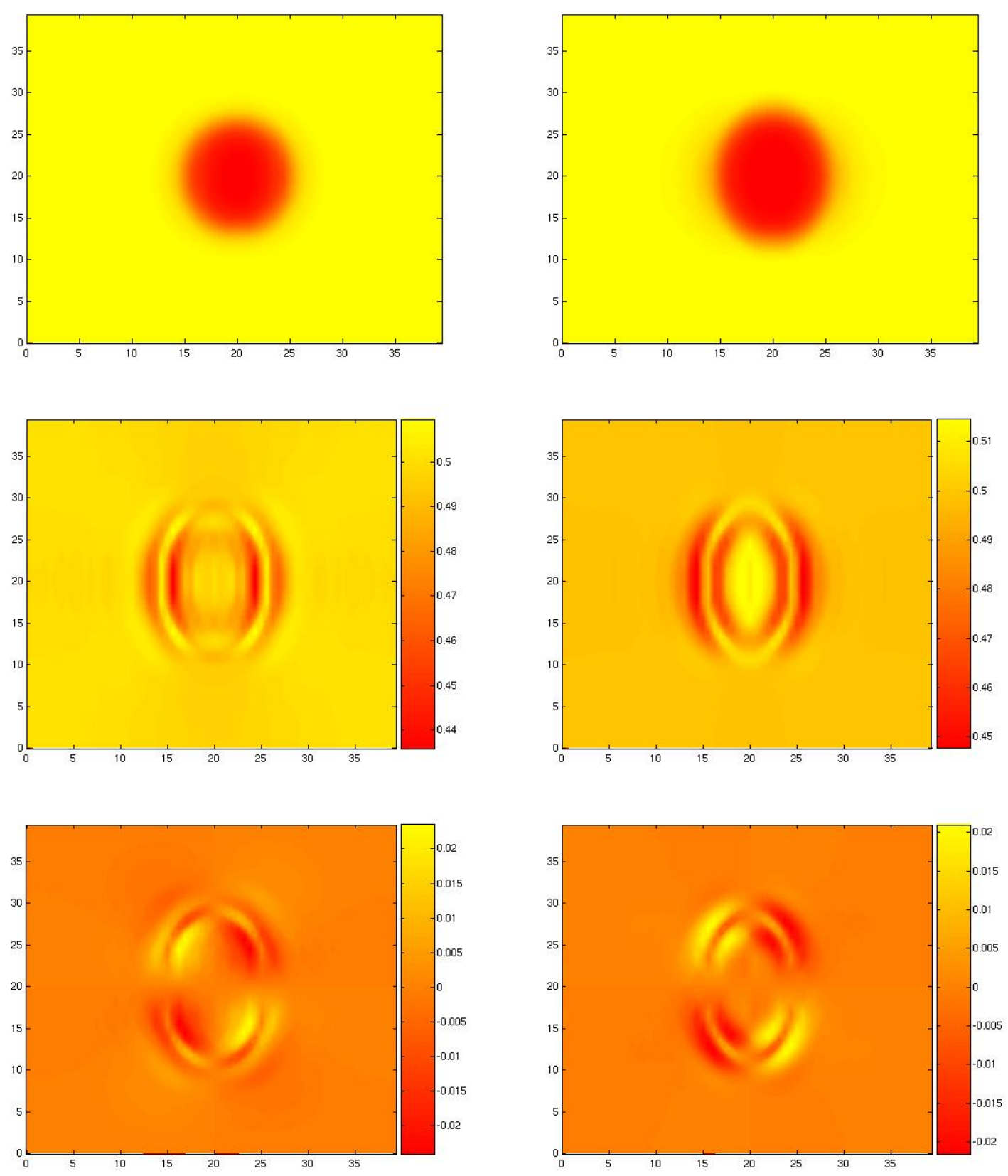

Fig. 3. Contour plots at time $t=150$ of $\rho_{a}$ (top), $\sigma_{11_{a}}+\sigma_{11_{b}}$ (middle), and $\sigma_{12_{a}}+\sigma_{12_{b}}$ (bottom). The left corresponds to $\xi_{a 0}=10, \xi_{b 0}=0.1, \tau_{a}=10, \tau_{b}=0.1$ and the right corresponds to $\xi_{a 0}=0.1, \xi_{b 0}=10, \tau_{a}=0.1, \tau_{b}=10$. On the left, due to the greater mobility and shorter stress relaxation time of the fluid in the droplet (polymer b), the droplet has formed a circular shape (minimizing the length of the interface) and has decreased in area from $146 R g^{2}$ to $101 R g^{2}$. On the right, the fluid in the droplet has less mobility and a longer stress relaxation time. Thus the droplet still has an elliptical shape and fills a larger area of the domain $\left(130 R g^{2}\right)$. In the plots of the stress, we observe that the droplet on the left has less bulk stress (middle left) and shear stress around the fluid interface (lower left) in comparison to the droplet on the right. 

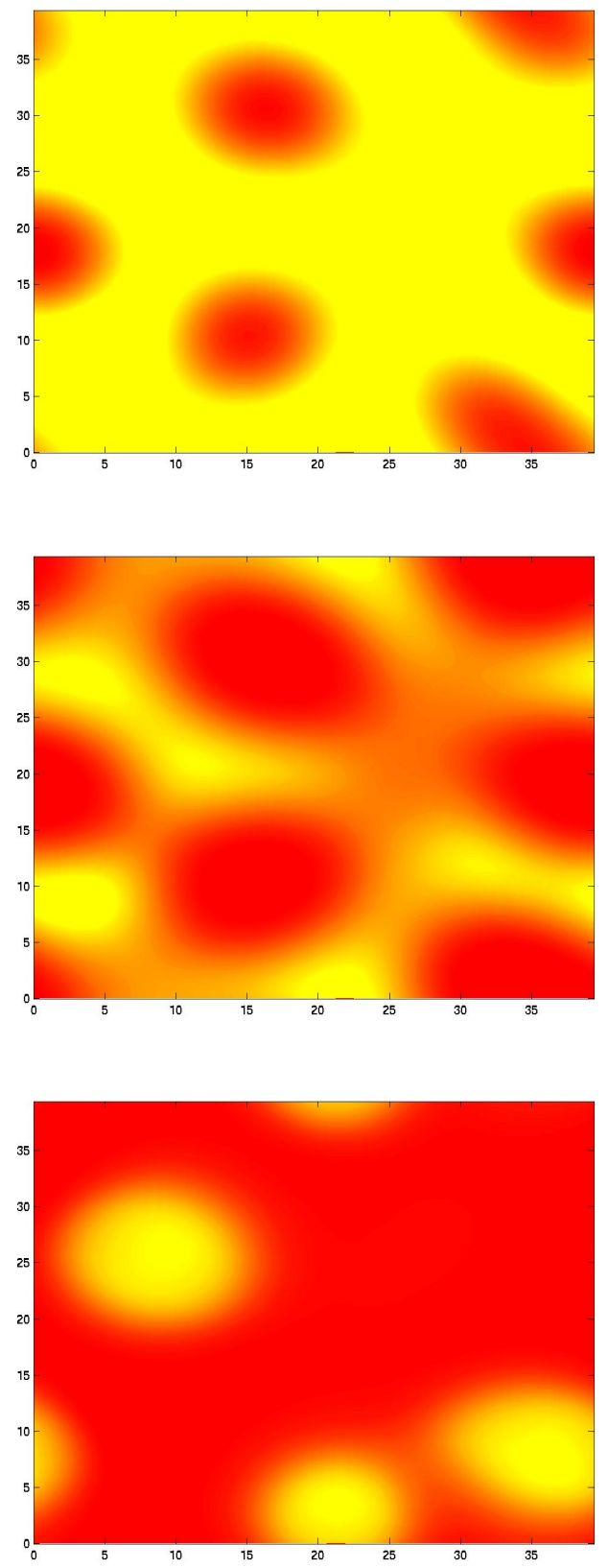

Fig. 4. Contour plots of $\rho_{a}$ (yellow) at times $t=2900$ (top), $t=3480$ (middle), and $t=4000$ (bottom). Initially, polymer $b$ has greater mobility and nucleates into droplets, even though it is the majority phase (top plot). Next the droplets grow and a network is formed by the polymer $a$ molecules (middle plot). Finally, the network breaks down and phase inversion occurs (bottom plot). 